\title{
Expression of Glucocorticoid Receptor and Early Growth Response Gene 1 during Postnatal Development of Two Inbred Strains of Mice Exposed to Early Life Stress
}

\author{
Sylvia Navailles ${ }^{a}$ Ross Zimnisky ${ }^{b} \quad$ Claudia Schmauss $^{a, b}$ \\ a Department of Molecular Therapeutics, New York State Psychiatric Institute, and b Department of Psychiatry, \\ Columbia University, New York, N.Y., USA
}

\section{Key Words}

Antidepressant treatment $\cdot$ Early growth response gene $1 \cdot$ Early life stress - Frontal cortex · Glucocorticoid receptor • Hippocampus • Postnatal development, mouse

\begin{abstract}
Early life stress can elicit profound changes in adult gene expression and behavior. One consequence of early life stress is a decreased expression of glucocorticoid receptors (GRs) in the frontal cortex and hippocampus. However, neither the time of onset nor the mechanism(s) leading to decreased GR expression during postnatal development are known. The present study used two inbred strains of mice that differ in their behavioral responsiveness to stress (Balb/c and C57BI/6), exposed them to an established paradigm of early life stress (infant maternal separation), and measured their expression of frontal cortical and hippocampal GRs and the putative transcriptional activator of the GR gene, early growth response gene (egr)-1, at defined stages of postnatal development. In both strains, real-time RT-PCR experiments revealed that decreased expression of GR in adolescence and adulthood is, in fact, preceded by increased GR expression during early life stress exposure. Thus, the early life
\end{abstract}

stress-induced disruption of the normal stress-hyporesponsive period during infancy is accompanied by increased GR expression. Moreover, chronic treatment with the antidepressant drug fluoxetine during adolescence or adulthood reversed the effect of early life stress on adult GR mRNA expression. In contrast to the strain-independent effect of early life stress on GR expression, however, changes in egr-1 expression occurred only in Balb/c mice, and unlike the biphasic developmental changes in GR mRNA expression, egr-1 mRNA was decreased throughout postnatal development. Moreover, there was no consistent overlap of anatomic regions affected by decreased GR and egr- 1 protein expression. Thus, in Balb/c mice, changes in GR and egr-1 expression can independently contribute to the phenotypes resulting from early life stress exposure. These findings illustrate that the impact of early life stress on gene expression changes is modulated by the genetic background and that the persistent changes in GR and egr-1 expression that arise early during postnatal developmental are reversible by chronic fluoxetine treatment during adolescence and adulthood.

Copyright $\odot 2010$ S. Karger AG, Basel

\section{KARGER \\ Fax +4161306 1234 \\ E-Mail karger@karger.ch}

www.karger.com (c) 2010 S. Karger AG, Basel

Accessible online at: www.karger.com/dne
Claudia Schmauss

Department of Psychiatry, Columbia University

Box 62, 1051 Riverside Drive

New York, NY 10032 (USA)

Tel. +1 212543 6505, E-Mail cs581@ columbia.edu 


\section{Introduction}

Early life stress can exert profound effects on adult emotional behavior and increases the risk for mood and anxiety disorders [Holmes et al., 2005]. Clinical studies identified a dysregulated hypothalamic pituitary adrenal (HPA) axis as a trait marker for vulnerability to depression [Ribeiro et al., 1993; Holsboer et al., 1995]. The HPA axis is tightly regulated by glucocorticoid inhibition via two corticosteroid receptors, the mineralocorticoid receptor (MR) and the glucocorticoid receptor (GR). Whereas the MR has high affinity for glucocorticoids and mediates tonic feedback to the HPA axis, the GR has lower ligand affinity and is primarily occupied following acute stress exposure [de Kloet et al., 1998]. The GRs located in the prefrontal cortex (PFC) and hippocampus are key mediators of the negative feedback regulation of the mature HPA axis [Mizoguchi et al., 2003].

Many studies have used animal models to examine the response of the HPA axis to stress. In the rodent, for example, corticosterone levels are low between postnatal day 2 (P2) and P14 and, during this period, stimuli that normally increase corticosterone levels do not lead to the activation of the HPA axis. This is known as the stresshyporesponsive period (SHRP), which is strongly influenced by maternal behavior [Levine, 1994] and thought to be due to pituitary GRs that exert a suppressive effect on the HPA axis [Schmidt et al., 2009]. During the SHRP, the number of GRs is only $50 \%$ of that found in the adult brain [Levine, 1994], and although the SHRP terminates at P14, the GR-mediated negative feedback occurs much later in development, as indicated by findings that the corticosterone response to an acute stressor is still higher, more prolonged, and less habituated during preadolescence than adulthood [McCormick et al., 2009].

In rodents, early life stress disrupts this SHRP, i.e. it disinhibits the response of the HPA axis to stress [Plotky and Meaney, 1993; Levine, 1994]. Early life stress also leads to decreased GR expression in the frontal cortex and hippocampus as well as decreased negative feedback inhibition of the HPA axis response to stress in adulthood [Ladd et al., 2004]. At present, however, neither the time of onset nor the mechanism(s) leading to decreased GR expression are fully understood. One possible mechanism could involve the zinc finger transcription factor early growth response gene (egr)-1, which belongs to a family of plasticity-inducible transcription factors [O'Donovan et al., 1999]. The induced expression of egr-1 has been linked to GR activation under acute stress conditions [Revest et al., 2005]. Moreover, a promotor region adjacent to exon
$1_{7}$ of the GR gene, whose activity in the hippocampus has been shown to be modulated by neonatal handling, contains a consensus sequence binding site for egr-1 [McCormick et al., 2000]. Also, there is some evidence that egr-1 binding and transcriptional activation is modulated by the methylation status of the $1_{7}$ promotor region [Weaver et al., 2007]. Since early life stress also leads to persistent changes in the expression of several other genes, it is possible that egr-1 expression is also affected. We therefore measured the expression of GR and egr-1 during the postnatal development of mice exposed to early life stress and also tested whether adolescent or adult treatment with the antidepressant fluoxetine would alter GR and egr-1 expression in adulthood. Moreover, although environmental factors can strongly affect the development of emotional behavior, the effect of genetic factors that determine the risk for developing adult psychopathology varies across mouse strains [Holmes et al., 2005]. Therefore, we examined two genetically distinct strains $(\mathrm{C} 57 \mathrm{Bl} / 6$ and $\mathrm{Balb} / \mathrm{c}$ ) that differ in 'emotionality' as assessed by their differences in sensitivity to early life and adult stress [Millstein and Holmes, 2007].

\section{Method}

\section{Animals}

All experiments involving animals were performed in accordance with the National Institutes of Health Guide for the Care and Use of Laboratory Animals and approved by the Institutional Animal Care and Use Committees at Columbia University and the New York State Psychiatric Institute. Male and female Balb/cJ and C57Bl/6J mice were housed in a facility with a 12-hour light/dark schedule (lights on at 6:00 AM) with free access to food and water.

\section{Infant Maternal Separation}

Offspring of first-time mothers were subjected to an infant maternal separation (IMS) paradigm, as previously described [Bhansali et al., 2007]. Pups were separated from their dam daily for $3 \mathrm{~h}$ (from 1:00 to 4:00 PM), starting at P2 and terminating at P15. Control animals were standard-facility-reared (SFR) offspring of first-time mothers. After weaning at P28, animals randomly selected from different litters were group housed by sex (4-5 animals per cage).

\section{RNA Extraction and Quantitative RT-PCR}

Animals were killed by rapid decapitation between 1:00 and 3:00 PM and their brains were removed. RNA was extracted from dissected forebrain neocortical tissue using guanidine/cesium chloride ultracentrifugation and used as a template for cDNA synthesis. The cDNA was amplified by real-time PCR to determine expression levels of GR and egr-1 mRNA. Synthetic 18-mer oligonucleotide primers specified the amplification of GR $\left(5^{\prime}\right.$ TGGAGCTACAGTCAAGGT-3' and 5'-AGCCGAAAGTCTGTTTCC-3') and egr-1 cDNA (5'-AAGGCCGAGATGCAATTG-3' 
and $5^{\prime}$-TATTCAGAGCGATGTCAG-3'). These primers were designed to amplify the $\mathrm{N}$-terminal regions of both open reading frames (approximately 200 base pairs) and to fit a single PCR protocol. The PCR products were run on agarose gels to ensure the existence of a single product. In real-time PCR experiments [performed with iQSYBR green supermixes (Bio-Rad, Hercules, Calif., USA)], measurements of the number of cycles required to reach the threshold fluorescence intensity (cycle threshold, Ct) were obtained. Ct values for each reaction were subtracted from Ct values of SFR animals that served as a baseline, and the result was referred to as $\Delta \mathrm{Ct}$. Fold changes in gene expression were calculated as $2^{\Delta \mathrm{Ct}}$. In all experiments, results were normalized to those obtained for amplifications of the same cDNA samples using primers designed to amplify $\beta$-actin cDNA because these $C t$ values did not differ between groups.

\section{Chronic Drug Treatments}

Fluoxetine hydrochloride was administered chronically via the drinking water either during adolescence (P32-P59) or adulthood (P60-P84). The drug was dissolved in water, and mice consumed approximately $16 \mathrm{mg} / \mathrm{kg}$ of fluoxetine per day. This dose has been shown to yield 'therapeutic' plasma levels [Dulawa et al., 2004], and we have previously shown significant effects of this dose on behavior and gene expression in mice [Bhansali et al., 2007; Navailles et al., 2008].

\section{Tissue Preparation for Immunocytochemistry}

Mice were anesthetized with ketamine $(100 \mathrm{mg} / \mathrm{kg})$ and xylazine $(15 \mathrm{mg} / \mathrm{kg})$ between 1:00 and 3:00 PM and perfused transcardially with $4 \%$ paraformaldehyde in $0.1 \mathrm{M}$ phosphate buffer (PB; $\mathrm{pH}$ 7.4). Brains were removed, postfixed for $1 \mathrm{~h}$ in the same fixative, and cryoprotected overnight in $0.1 \mathrm{M} \mathrm{PB}$ containing $30 \%$ sucrose at $4^{\circ} \mathrm{C}$. For each brain, a series of adjacent, coronal $40-\mu \mathrm{m}-$ thick microtome sections were collected in six representative sets (240 $\mu \mathrm{m}$ apart) from bregma 2.8 to $-4 \mathrm{~mm}$ [Hof et al., 2000]. Sections were processed either for egr-1 or GR immunostaining. Adjacent sections were counterstained with thionine to clarify the cytoarchitecture.

\section{Immunohistochemistry}

After inhibition of endogenous peroxidase activity (a 30-min incubation with $0.3 \% \mathrm{H}_{2} \mathrm{O}_{2}$ in $\mathrm{PB}$ at room temperature), freefloating sections were blocked for $1 \mathrm{~h}$ with $0.5 \%$ bovine serum albumin (BSA) prior to an overnight incubation at $4{ }^{\circ} \mathrm{C}$ with the primary antibody diluted in PB with $0.1 \%$ BSA and $0.25 \%$ Triton $\mathrm{X}-100$. Two polyclonal primary antibodies were used: a rabbit anti-egr-1 (C19) antibody (sc-189; 1:1,000; Santa Cruz Biotechnology, Santa Cruz, Calif., USA) and a rabbit anti-GR antibody (PA1511A; 1:75; Affinity BioReagents, Golden, Colo., USA). The affinity-purified anti-egr-1 antibody was raised against a peptide mapping at the carboxyl terminus of human/mouse egr-1. On Western blots, this antibody recognizes a single protein band of $80 \mathrm{kDa}$ and it does not cross-react with other members of the EGR family [DeSteno and Schmauss, 2008]. The anti-GR antibody was raised against an amino-terminal peptide of human GR (amino acids 346-367) and has been shown to cross-react with mouse GR, to detect a single $97-\mathrm{kDa}$ protein on Western blots, and to recognize all forms of GR protein (liganded, unliganded, or associated with DNA fragments) [Cidlowski et al., 1990]. Moreover, in immunocytochemical studies, GR immunoreactivity localized to the cytoplasm in the absence of a ligand, but became predominantly nuclear after hormone treatment [Cidlowski et al., 1990].

After incubation with primary antibody, sections were washed once in $\mathrm{PB}$ with $0.25 \%$ Triton $\mathrm{X}-100$, twice in pure $\mathrm{PB}$, and then incubated for $1 \mathrm{~h}$ at room temperature with biotinylated secondary antibody [biotin-conjugated goat anti-rabbit IgG (1:400 in PB containing 0.1\% BSA; Vector Laboratories, Burlingame, Calif., USA)]. After further washes in $\mathrm{PB}$, sections were incubated in avidin-biotin-peroxidase complex for $1 \mathrm{~h}$ (Vectastain Elite kit, 1:100 in PB; Vector Laboratories), followed by incubation for 5 min in $0.022 \% 3,3^{\prime}$-diaminobenzidine (DAB; Sigma) and $0.003 \%$ hydrogen peroxide in $\mathrm{PB}$. All sections were rinsed in $\mathrm{PB}$, mounted on gelatin-coated slides, air-dried, and coverslipped.

\section{Image Acquisition and Analysis}

Immunolabeled sections were viewed either at $\times 10$ or $\times 20$ magnification and captured using a Zeiss Axioskop 2 microscope equipped with a Zeiss Axiocam HRc r1.4 camera (Zeiss, Oberkochen, Germany). The microscopic images were photodocumented using Improvision Openlab 3.1 software (Improvision, Coventry, UK) and then processed using Adobe Photoshop (version CS3). All images were acquired in the same light conditions. Adjustments to image brightness and contrast were made for clarity of publication, but the resultant images reflect the experimental data.

The expression of egr-1 and GR immunoreactivity was analyzed using the public domain NIH ImageJ software (available at http://rsb.info.nih.gov/) by measuring the pixel densities in the brain regions of interest in three consecutive sections per animal. The digitized images were captured at bregma $-1.80,-2.04$, and $-2.28 \mathrm{~mm}$ to adequately cover the area of the hippocampus. The images (RGB color) were first converted to 8-bit grey scale and then calibrated using the pixel inverter function. Each region of interest was delineated, and the mean pixel density of the area selected was measured. To avoid variations in background labeling, which may affect measurements, and in order to obtain the specific pixel density value of egr-1 and GR labeling, the mean pixel density value of the background area surrounding the area of interest was systematically subtracted. Data represent the average values obtained for each hippocampal subregion in three consecutive sections, and they are expressed as the mean pixel density per region and animal group.

\section{Statistical Analysis}

For comparisons of gene expression changes between IMS mice and their controls during defined postnatal ages, two-tailed Student's $t$ tests were used. All other data were compared by one-way analysis of variance (ANOVA), and significant differences were resolved post hoc using the Tukey-Kramer multiple comparison test.

\section{Results}

\section{Expression of Forebrain Neocortical GR mRNA during Postnatal Development of IMS Balb/c and C57Bl/6 Mice}

We compared the expression of mRNA encoding GR during the postnatal development of IMS mice and their SFR controls using real-time PCR. As shown in figure 1, 


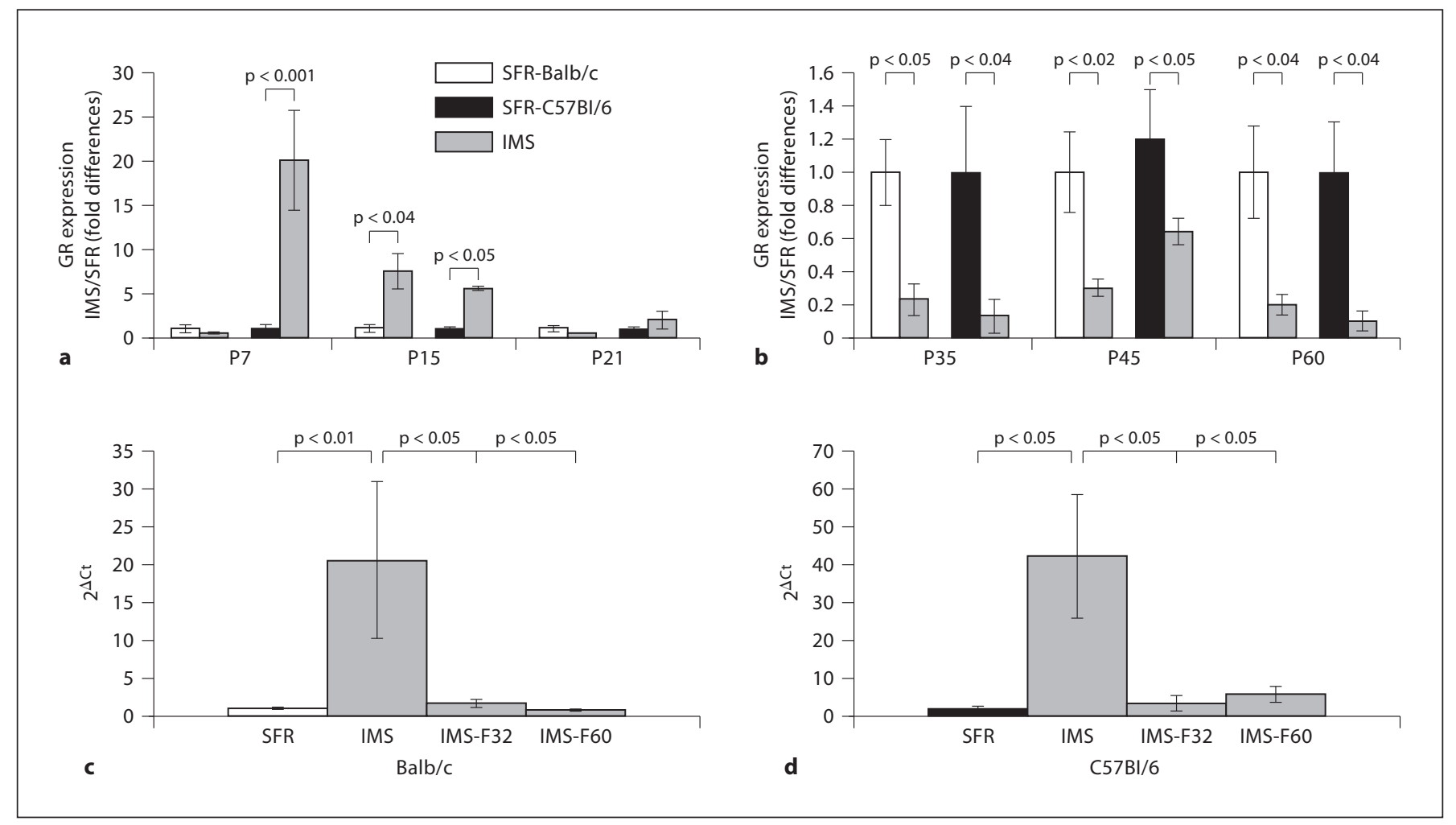

Fig. 1. Biphasic changes in the expression of forebrain neocortical mRNA encoding GR during postnatal development of Balb/c and C57Bl/6 mice exposed to IMS. a Both strains exhibited increased GR expression on the last day of IMS exposure (P15) and, in C57Bl/6 mice, increased GR expression was already detected at P7. b There was a significant decrease in GR expression in both strains starting at P35 and persisting into adulthood (P60). a, $\mathbf{b}$ Means \pm SEM of fold changes (relative to SFR controls) determined for 6-8 animals per group (two-tailed Student's t test). c In Balb/c mice, chronic fluoxetine treatment $(16 \mathrm{mg} / \mathrm{kg} /$ day $)$ of

IMS mice during adolescence (P32-P59, labeled IMS-F32) or adulthood (P60-P84, labeled IMS-F60) completely normalized GR expression levels as shown here with the actual $2^{\Delta C t}$ values of the real-time PCR measures. Data are means \pm SEM from 6 animals per group and were compared by one-way ANOVA: $\mathrm{F}(3,20)=5.93 ; \mathrm{p}=0.005$. $\mathbf{d}$ In IMS C57Bl/6 mice, adolescent and adult fluoxe-tine treatment also normalized GR expression levels [ANOVA, $\mathrm{F}(3,19)=5.12, \mathrm{p}=0.009 ; \mathrm{n}=6$ animals per group]. c, d Statistical differences revealed by ANOVA were resolved post hoc (Tukey-Kramer multiple comparison tests) as indicated.

IMS Balb/c and C57Bl/6 mice exhibited similar biphasic changes in GR expression. In both strains, GR expression was increased during and/or at the end of the IMS relative to SFR controls. In C57Bl/6 mice, this increase was significant at P7 and P15, and in Balb/c mice, increased GR expression was significant at P15 (fig. 1a). At P21, however, GR expression levels were indistinguishable between SFR controls and IMS mice (fig. 1a). Also, in both strains, decreased expression of GR was detected at P35 and persisted into adulthood (fig. 1b).

As further shown in figure $1 \mathrm{c}$ and $1 \mathrm{~d}$, in both strains, the decrease in adult (P60 and P85) GR expression was completely reversed by chronic treatment with the antidepressant fluoxetine administered during either adolescence (P32-P59) or adulthood (P60-P84). This effect of

fluoxetine on GR expression was specific to IMS mice since additional experiments on SFR Balb/c mice treated with fluoxetine during adolescence and adulthood revealed that their GR expression levels were indistinguishable from non-treated SFR mice: fold differences (SFR/ SFR-fluoxetine): $1.15 \pm 0.21$ after adolescent and $0.98 \pm$ 0.09 after adult treatment.

In contrast to the biphasic changes in GR expression in IMS mice, the expression levels of mRNA encoding the MR were unchanged in both strains, with only one exception: just as P7 IMS C57Bl/6 mice expressed dramatically ( $\sim 20$-fold) higher levels of GR (fig. 1a), they also expressed about 8-fold higher levels of MR at this age compared with SFR controls (table 1). However, at all other developmental ages, the expression levels of MR were in- 
Table 1. Real-time PCR measurements of MR mRNA expression levels in the forebrain neocortex of SFR and IMS Balb/c and C57Bl/6 mice during postnatal development

\begin{tabular}{llll}
\hline & & SFR & IMS \\
\hline P7 & Balb/c & $0.83 \pm 0.2$ & $0.83 \pm 0.3$ \\
& C57B1/6 & $1.79 \pm 0.43$ & $0.8 \pm 0.26^{*}$ \\
P15 & Balb/c & $0.95 \pm 0.36$ & $0.85 \pm 0.34$ \\
& C57B1/6 & $0.67 \pm 0.39$ & $1.16 \pm 0.5$ \\
P21 & Balb/c & $0.68 \pm 0.22$ & $0.61 \pm 0.21$ \\
& C57B1/6 & $0.59 \pm 0.29$ & $0.86 \pm 0.34$ \\
P35 & Balb/c & $0.90 \pm 0.10$ & $1.37 \pm 0.49$ \\
& C57B1/6 & $1.27 \pm 0.51$ & $0.85 \pm 0.17$ \\
P45 & Balb/c & $0.58 \pm 0.10$ & $0.88 \pm 0.33$ \\
& C57B1/6 & $0.49 \pm 0.15$ & $0.84 \pm 0.15$ \\
P60 & Balb/c & $0.71 \pm 0.12$ & $0.41 \pm 0.14$ \\
& C57B1/6 & $0.56 \pm 0.25$ & $0.59 \pm 0.24$ \\
\hline
\end{tabular}

${ }^{*} \mathrm{p}<0.03$ vs. SFR. Data are $2^{\Delta \mathrm{Ct}}$ values and statistical differences were determined with two-tailed Student's t tests. distinguishable between SFR and IMS mice (table 1). As a result, in both strains, IMS led to altered MR/GR mRNA expression ratios at P15, P45, and P60 (table 2).

In summary, in IMS mice of two genetically different strains, changes in GR mRNA expression were biphasic: expression was increased during infancy but decreased in adolescence and adulthood. Chronic treatment with the antidepressant fluoxetine reversed this effect in adult animals, regardless of whether the treatment was initiated during adolescence or adulthood.

Expression of Forebrain Neocortical egr-1 $m R N A$ during Postnatal Development of IMS Balb/c and C57Bl/6 Mice

In contrast to the changes in GR expression that are common to both strains of mice, egr-1 mRNA expression levels of IMS C57Bl/6 mice did not significantly differ from SFR controls at all postnatal ages (fig. 2a). In IMS $\mathrm{Balb} / \mathrm{c}$ mice, however, significantly decreased egr-1 mRNA expression was detected at all developmental ages examined (fig. 2b). Moreover, chronic treatment with fluoxetine during adolescence and adulthood had no significant effect on egr-1 mRNA expression in IMS C57Bl/6 mice (fig. 2c). It did, however, reverse the effect of IMS on egr-1 expression in Balb/c mice (fig. 2d). Moreover, the magnitude of increased egr-1 expression in IMS Balb/c mice treated with fluoxetine was larger than the increase in egr-1 mRNA expression detected in SFR Balb/c mice
Table 2. Ratios of MR/GR mRNA expression in the forebrain neocortex of SFR and IMS Balb/c and C57Bl/6 mice during postnatal development

\begin{tabular}{llcrl}
\hline \multicolumn{4}{l}{ SFR } & \multicolumn{1}{l}{ IMS } \\
\hline P7 & Balb/c & $7.6 \pm 2.9$ & $12.8 \pm 4.4$ & \\
& C57B1/6 & $1.3 \pm 0.1$ & $2.8 \pm 0.9$ & \\
P15 & Balb/c & $20.5 \pm 9.4$ & $3.7 \pm 1.0$ & $\mathrm{p}<0.05$ \\
& C57B1/6 & $55.8 \pm 17.7$ & $6.18 \pm 3.5$ & $\mathrm{p}<0.04$ \\
P21 & Balb/c & $21.5 \pm 8.5$ & $32.1 \pm 5.4$ & \\
& C57B1/6 & $27.8 \pm 7.9$ & $24.8 \pm 9.8$ & \\
P35 & Balb/c & $0.6 \pm 0.2$ & $1.1 \pm 0.5$ & \\
& C57B1/6 & $1.1 \pm 0.6$ & $2.8 \pm 0.8$ & \\
P45 & Balb/c & $5.0 \pm 2.2$ & $21.5 \pm 9.6$ & $\mathrm{p}<0.05$ \\
& C57B1/6 & $1.9 \pm 0.4$ & $5.4 \pm 1.9$ & $\mathrm{p}<0.05$ \\
P60 & Balb/c & $5.3 \pm 1.7$ & $31.4 \pm 10.3$ & $\mathrm{p}<0.03$ \\
& C57B1/6 & $2.6 \pm 1.6$ & $20.9 \pm 10.9$ & $\mathrm{p}<0.05$ \\
\hline
\end{tabular}

Statistical differences were determined with two-tailed Student's t tests. treated with fluoxetine during adolescence and adulthood [fold differences (SFR/SFR-fluoxetine): $1.8 \pm 0.11$ after adolescent $(\mathrm{p}<0.04)$ and $1.6 \pm 0.27$ after adult treatment $(p<0.05)]$. Nevertheless, the effects of adolescent and adult fluoxetine treatment in SFR mice were also unique to $\mathrm{Balb} / \mathrm{c}$ mice since the corresponding treatment of SFR C57Bl/6 mice did not alter egr-1 expression levels [fold differences (SFR/SFR-fluoxetine): $1.11 \pm 0.23$ (adolescent treatment) and $1.31 \pm 0.17$ (adult treatment)].

Hence, in contrast to the changes in GR expression detected in both strains, changes in egr-1 expression following IMS do not follow the biphasic postnatal developmental pattern detected for GR expression levels. Moreover, the effect of chronic fluoxetine treatment on forebrain neocortical egr-1 mRNA expression levels differs between both strains.

\section{Comparison of Hippocampal GR and egr-1 Protein \\ Expression in SFR and IMS Balb/c Mice}

To further test whether the persistent decrease in GR expression from mid-adolescence (P35) to young adulthood (P60) detected in the forebrain neocortex also occurs in the hippocampus, we performed additional immunocytochemical studies on IMS and SFR mice that allowed us to compare GR protein expression between all hippocampal subfields. Moreover, we chose Balb/c mice for these studies because only these mice allowed us to also examine whether there is an overlap in anatomic re- 


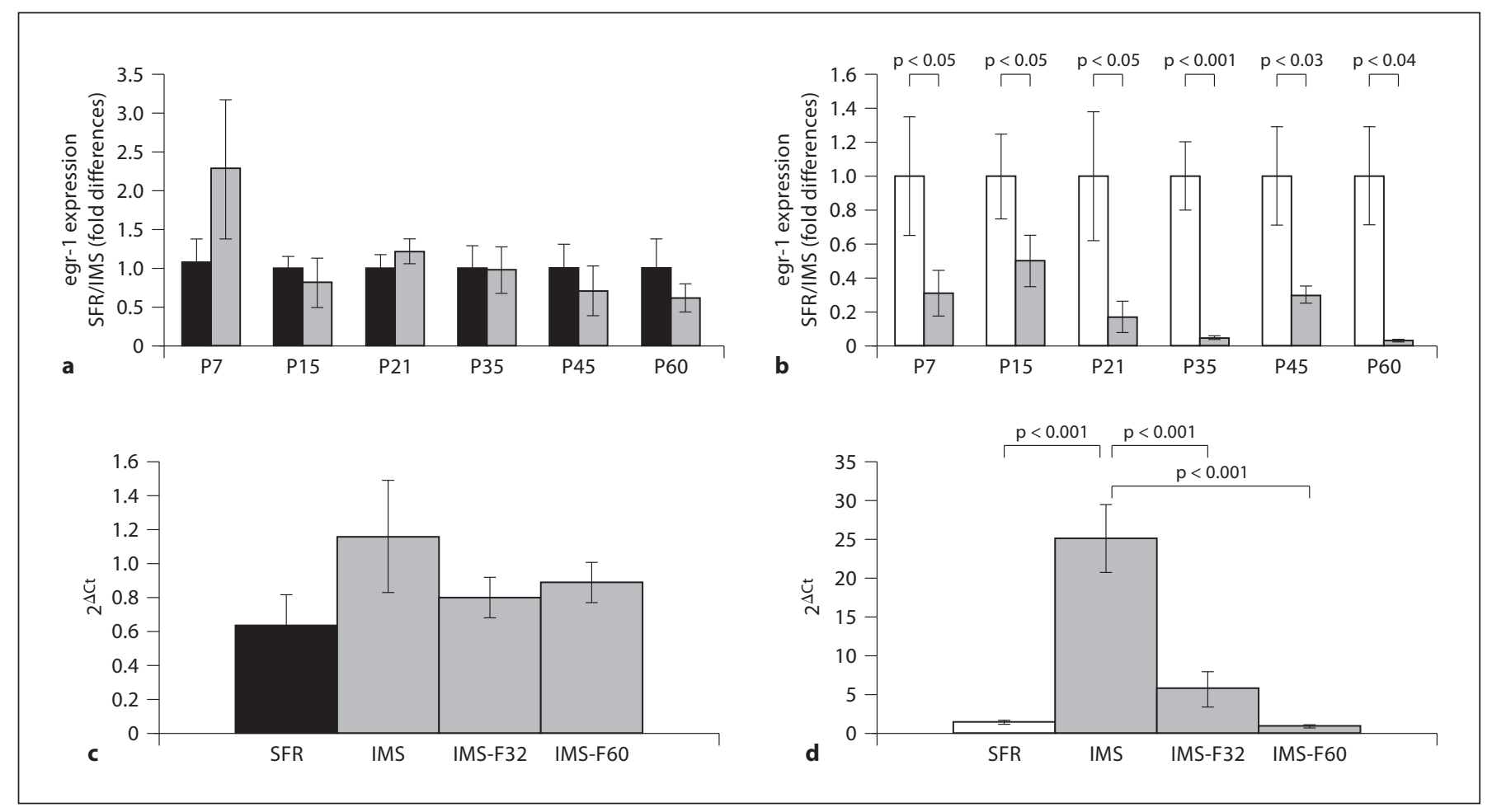

Fig. 2. Differential effects of IMS on the expression of forebrain neocortical egr-1 mRNA in Balb/c and C57Bl/6 mice. a egr-1 mRNA expression in SFR (black bar) and IMS C57Bl/6 mice (grey bars) during postnatal development measured with real-time PCR. b Corresponding measures of egr-1 mRNA expression in SFR (white bar) and IMS Balb/c mice (grey bars). a, b Data are means \pm SEM $(n=5-7)$ and were compared by Student's $t$ test. c Comparison of real-time PCR cycle thresholds (expressed as
$2^{\Delta \mathrm{Ct}}$ ) for egr-1 amplification in IMS C57Bl/6 mice treated with fluoxetine during adolescence (IMS-F32) or adulthood (IMSF60). One-way ANOVA $[\mathrm{F}(3,12)=1.066 ; \mathrm{p}=0.4]$ revealed no significant differences between the four animal groups. d Corresponding comparison of $2^{\Delta \mathrm{Ct}}$ values for egr-1 amplification in IMS Balb/c mice. Significant differences revealed by ANOVA $[\mathrm{F}(3,20)=26.2 ; \mathrm{p}=0.0001]$ were resolved post hoc using TukeyKramer multiple comparison tests. gions with decreased expression of GR and egr-1 proteins.

As shown in figure 3, in P35 IMS mice, GR expression was decreased in all hippocampal subfields. [Of note: in SFR mice, GR expression levels were significantly higher at P35 (dentate gyrus: $\mathrm{p}<0.01$; cornu ammonis, CA, subfields: $\mathrm{p}<0.001$ ) than at P60, but this elevated GR expression was not detected in P35 IMS mice]. In P60 IMS mice, however, hippocampal GR expression levels were indistinguishable from SFR controls (fig. 3). Thus, in contrast to the forebrain neocortex, decreased GR expression in the hippocampus was only transiently detected during mid-adolescence.

A different result was obtained when egr-1 immunolabeled sections were analyzed. In the hippocampus of P35 IMS Balb/c mice, egr-1 protein expression was decreased only in the dentate gyrus and the CA2 subfield, but increased in the CA1 subfield (fig. 4). At P60, de- creased egr-1 expression was still detected in the CA2 subfield, but was not seen in any other hippocampal subfields. Thus, in the hippocampus of Balb/c mice exposed to early life stress, there was no consistent overlap between anatomic subfields of decreased expression of GR and egr-1 protein.

\section{Discussion}

The present study shows that mice exposed to early life stress exhibit a biphasic change in GR mRNA expression. Our finding of decreased GR expression in the forebrain neocortex during later postnatal developmental stages of IMS mice is consistent with results of previous studies on adult rodents exposed to IMS [Ladd et al., 2004]. However, we also found increased GR mRNA expression at $\mathrm{P} 7 / \mathrm{P} 15$, a period known as the SHRP in non-stressed 


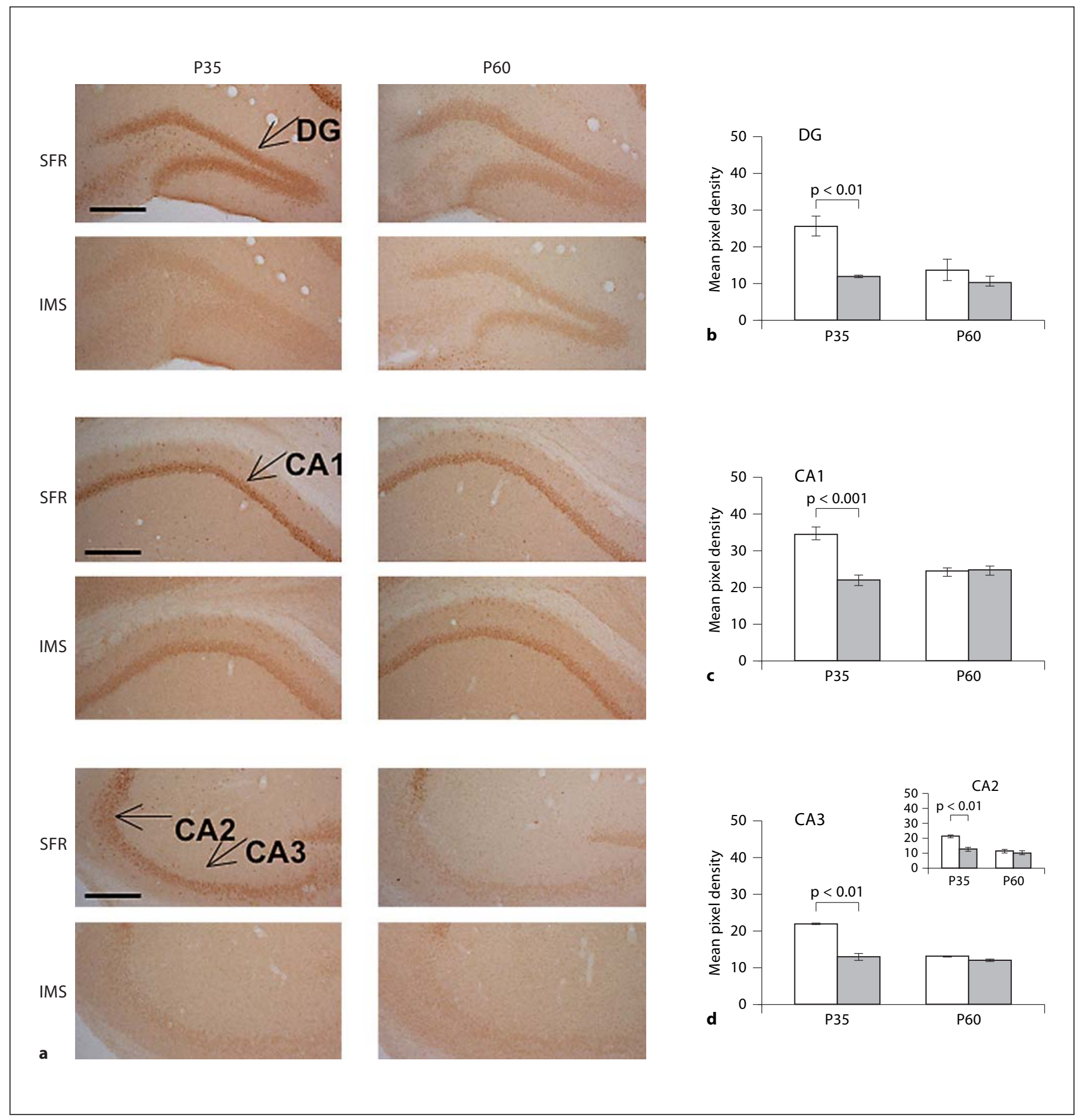

Fig. 3. Expression of GR protein in the hippocampus of SFR and IMS Balb/c mice at P35 and P60. a Representative images of DABstained sections labeled with an anti-GR antibody. The hippocampal subfields are indicated. DG = Dentate gyrus. b-d Comparison of pixel densities (means \pm SEM; measured for 4-6 animals per group) between SFR (white bars) and IMS (grey bars)
Balb/c mice. Statistical differences revealed by one-way ANOVA [DG: $\mathrm{F}(3,20)=8.478 ; \mathrm{CA} 1:(\mathrm{F} 3,20)=14.152 ; \mathrm{CA} 3: \mathrm{F}(3,20)=61.332$; $\mathrm{p}<0.001]$ were resolved post hoc using Tukey-Kramer multiple comparison tests as indicated. Note the significant decrease in GR expression in all four hippocampal subfields that is only evident at P35. 
P35

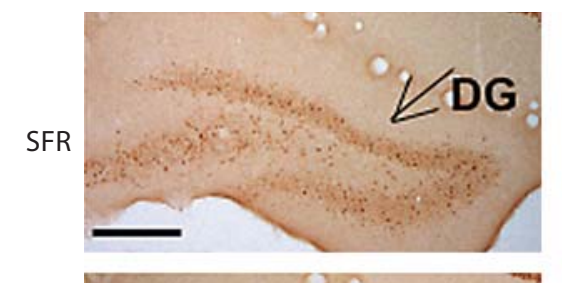

IMS

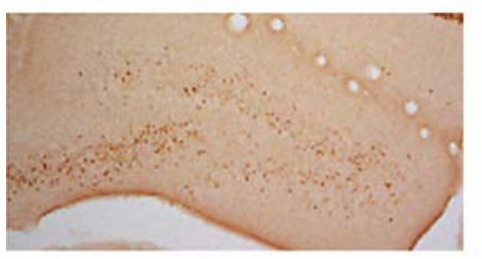

SFR

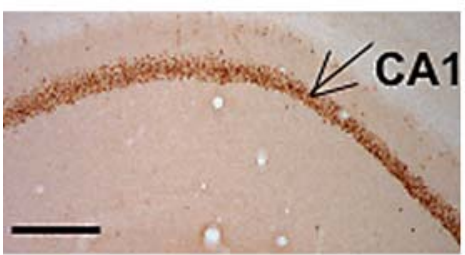

IMS

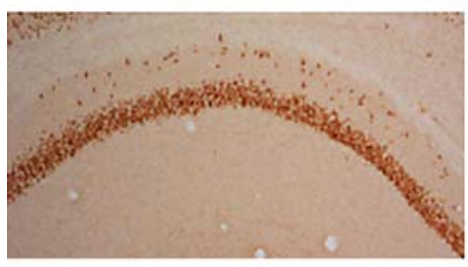

SFR

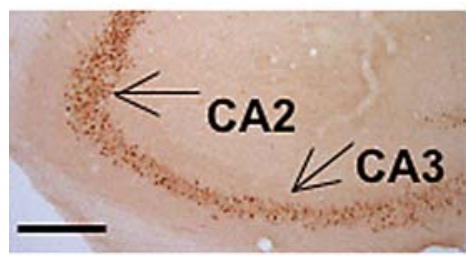

IMS

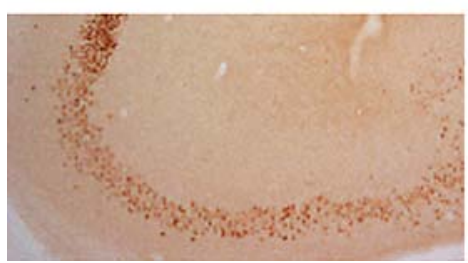

a
P60
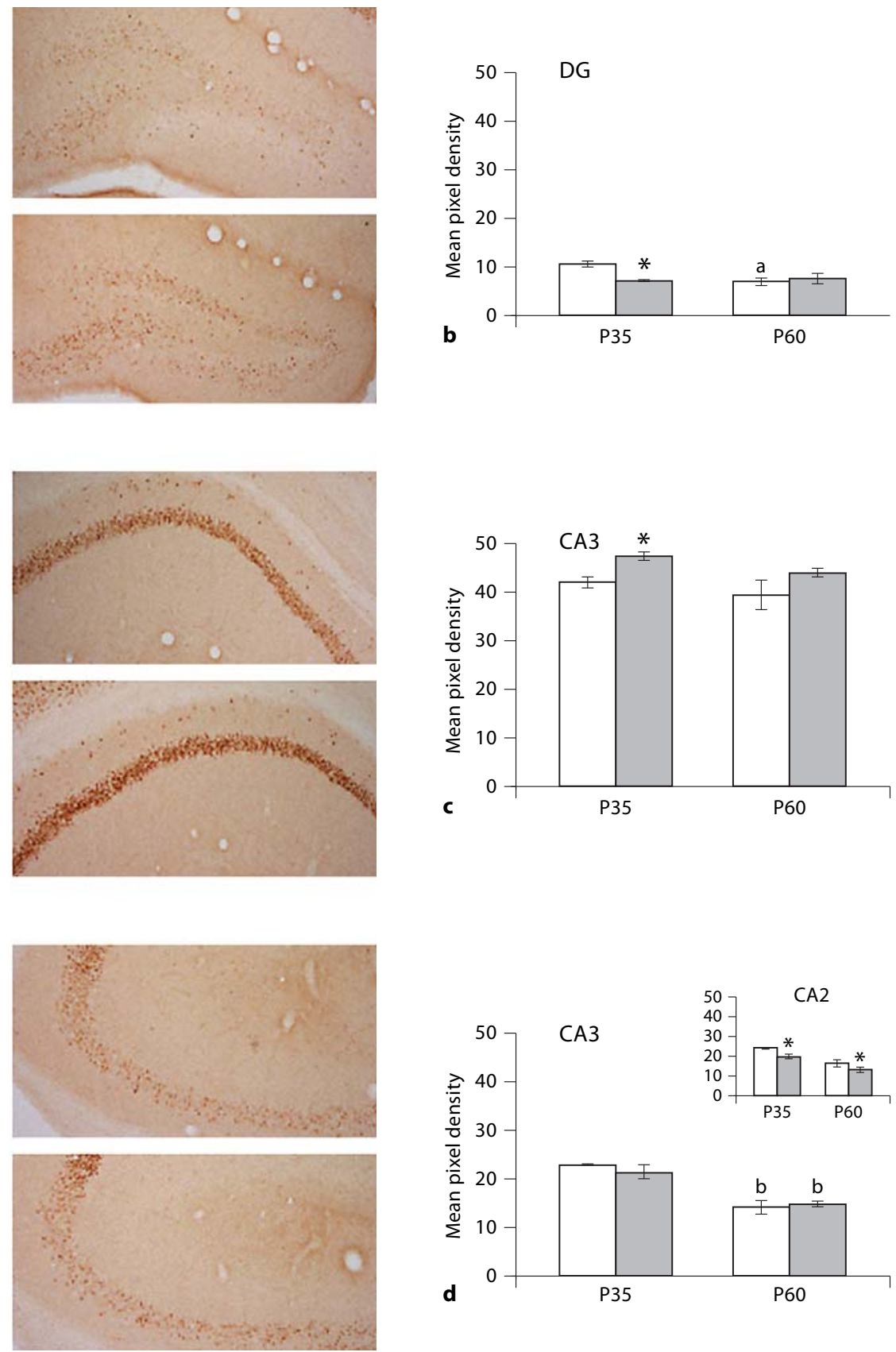

Fig. 4. Expression of egr-1 protein in the hippocampus of SFR and IMS Balb/c mice. a Representative images of hippocampal sections labeled with an antibody raised against egr-1. b-d Mean pixel density of egr-1 immunoreactivity in all hippocampal subfields. Data were obtained from 4-6 animals per group and compared by one-way ANOVA $[\mathrm{DG}$ : $\mathrm{F}(3,20)=3.134$; $\mathrm{p}<0.05$, CA1:
$\mathrm{F}(3,20)=3.245 ; \mathrm{p}>0.05$, CA2: $\mathrm{F}(3,20)=17.433, \mathrm{p}<0.001, \mathrm{CA} 3:$ $\mathrm{F}(3,20)=13.475 ; \mathrm{p}<0.001]$ followed post hoc by Tukey-Kramer multiple comparison tests. ${ }^{*} \mathrm{p}<0.05$ vs. SFR controls of the corresponding age; ${ }^{\mathrm{a}} \mathrm{p}<0.05$ vs. SFR $\mathrm{P} 35$ values and ${ }^{\mathrm{b}} \mathrm{p}<0.001$ vs. corresponding $\mathrm{P} 35$ values. 
mice. Compared with IMS Balb/c mice, the increase in GR expression occurred earlier in IMS C57Bl/6 mice which, at P7, expressed not only the highest levels of GR mRNA (a $\sim 20$-fold increase) but also showed an $\sim 8$-fold increase in MR mRNA. However, in C57Bl/6 mice, increased MR expression was no longer detected at P15 and was never noticed in IMS Balb/c mice. Although the reason for this early and robust increase in both GR and MR in P7 IMS C57Bl/6 mice is presently not known, the SHRP is thought to be strongly regulated by maternal behavior [Levine, 1994]. Thus, the present finding suggests that increased GR expression in IMS pups during the time of early life stress exposure is also a maternally induced effect and could enable an earlier onset of a negative feedback regulation of the response of the HPA axis to stress.

The mechanisms underlying the biphasic change in GR mRNA expression in response to early life stress are presently unknown. We show here that the levels of mRNA encoding GR, but not MR (with the exception of P7 IMS C57Bl/6 mice), are altered after early life stress exposure. Thus, although the ratios of MR/GR are significantly altered in IMS mice at P15, and at P45 and P60 when compared to SFR controls (table 2), these changes in the MR/GR ratio are solely due to changes in GR mRNA expression levels. Since MRs mediate tonic feedback to the HPA axis [de Kloet et al., 1998], it is not surprising that baseline corticosterone levels were found to be similar in IMS and SFR rats [Plotzky and Meaney, 1993]. However, one salient feature of the response to the HPA axis to acute stress in IMS animals is the prolonged elevation of plasma corticosterone levels due to decreased GR-mediated negative feedback regulation of the HPA axis [Plotzky and Meaney, 1993; Ladd et al., 2004]. In this regard, it is of interest to note that the changes in GR expression in IMS mice are specific for the age and brain region. While decreased GR protein expression was detected in the hippocampus of IMS Balb/c mice at P35, GR expression returned to normal at P60 (fig. 3). In contrast, neocortical GR mRNA remained expressed at decreased levels from P35 to P60 (fig. 1). It is thus important to note that GR expression in the PFC is thought to be the most prominent factor in determining stress responses of the HPA axis, particularly in animals exposed to early life stress [Ladd et al., 2004]. This is also supported by findings that, in the medial PFC and hippocampus, chronic adult stress differentially regulates the levels of cytosolic and nuclear GR, i.e. levels of cytosolic (non-activatable) GR were found to be lower in the PFC [Ladd et al., 2004].
Although decreased GR expression in IMS mice persisted from mid-adolescence into adulthood, this change in GR mRNA expression could be completely reversed by chronic treatment with the antidepressant fluoxetine during either adolescence (P32-P59) or adulthood (P60P84). Increased GR mRNA expression in fluoxetinetreated IMS mice, however, cannot simply be explained by the pharmacological effect of fluoxetine (a serotoninselective reuptake inhibitor) influencing GR expression, as the same fluoxetine treatment failed to alter GR expression in SFR mice. Thus, the mechanism(s) by which chronic fluoxetine increases GR mRNA expression in IMS mice remain to be elucidated.

Finally, whereas the changes in GR expression are common to both strains, decreased egr-1 expression, a putative transcriptional activator of the GR gene, was only detected in IMS Balb/c mice. In addition to this dependence upon the genetic background, the early postnatal developmental profile (P7/P15) of decreased egr-1 expression in IMS Balb/c mice, as well as the anatomic pattern of decreased egr-1 protein expression in hippocampal subfields during adolescence (P35) and adulthood (P60), did not overlap with the changes in GR expression detected in these mice. Thus, changes in GR expression are not simply due to altered transcriptional regulatory activity of egr-1, and decreased GR and egr-1 expression levels are likely to contribute independently to the phenotypes of IMS mice that result from early life stress exposure. In IMS Balb/c mice, decreased egr-1 expression could have significant consequences for other egr-1-induced plastic changes in gene expression, in particular those involved in higher cognitive functions [O'Donovan et al., 1999].

The changes in egr-1 expression in IMS Balb/c mice are also reversible by chronic treatment with fluoxetine during adolescence or adulthood. However, this effect can also not be explained by the direct effect of fluoxetine on egr-1 expression. First, despite the robust effect of fluoxetine in IMS Balb/c mice, fluoxetine had no effect on egr-1 expression in SFR and IMS C57Bl/6 mice. Second, in addition to the dependence upon the genetic background, the magnitude of the increase in egr-1 expression in fluoxetine-treated SFR Balb/c mice is substantially lower than that of fluoxetine-treated IMS Balb/c mice.

In conclusion, the study illustrates that, although some changes in gene expression resulting from early life stress are not dependent upon the genetic background, others are clearly due to a genetic disposition. The present findings illustrate that elucidating the impact of early life stress on adult psychopathology requires studying the in- 
teraction between the genetic background and stressful life events as well as the onset of abnormalities in postnatal development resulting from early life stress. Importantly, although we show here that the onset of persistent changes in gene expression following early life stress exposure occurs early during adolescent development, the changes in gene expression are nevertheless reversible, even in the adult brain.

\section{Acknowledgments}

This work was supported by a grant from the National Institutes of Health (RO1 MH078993 to C.S.) and the Whitehall Foundation (2007-12-77 to C.S.). S.N. was supported in part by a National Institutes of Health Conte Center grant P50 MH062185.

\section{References}

Bhansali P, Dunning J, Singer SE, David L, Schmauss C (2007): Early life stress alters adult serotonin $2 \mathrm{C}$ receptor pre-mRNA editing and expression of the $\alpha$ subunit of the heterotrimeric G-protein Gq. J Neurosci 27: 1467-1473.

Cidlowski JA, Bellingham DL, Powell-Oliver FE, Lubahn DB, Sar M (1990): Novel anti-peptide antibodies to the human glucocorticoid receptor: recognition of multiple receptor forms in vitro and distinct cytoplasmic and nuclear receptors. Mol Endocrinol 4:14271437.

de Kloet ER, Vreugdenhil E, Oitzl MS, Joels M (1998): Brain corticosteroid receptor balance in health and disease. Endocr Rev 19:269301.

DeSteno DA, Schmauss C (2008): Induction of early growth response genes 2 in the forebrain of mice performing an attention-setshifting task. Neuroscience 152:417-428.

-Dulawa S, Holick KA, Gundersen B, Hen R (2004): Effects of chronic fluoxetine in animal models of anxiety and depression. Neuropsychopharmacology 29:1321-1330.

Hof PR, Young WG, Bloom FE, Belichenko PV, Celio MR (2000): Comparative Cytoarchitectonic Atlas of the C57Bl/6 and 129/SV Mouse Brains. Amsterdam, Elsevier.

-Holmes A, le Guisquet AM, Vogel E, Millstein RA, Leman S, Belzung C (2005): Early life genetic, epigenetic, and environmental factors shaping emotionality in rodents. Neurosci Biobehav Rev 29:1335-1346.
Holsboer F, Lauer CJ, Schreiber W, Krieg JC Navailles S, Hof PR, Schmauss C (2008): Antide(1995): Altered hypothalamic-pituitary-adrenocortical regulation in healthy subjects at high familiar risk for affective disorders. Neuroendocrinology 62:340-347.

Ladd CO, Huot RL, Thrivikraman KV, Nemeroff CB, Plotsky PM (2004): Long-term adaptations in glucocorticoid receptor and mineralocorticoid receptor mRNA and negative feedback on the hypothalamo-pituitary-adrenal axis following neonatal maternal separation. Biol Psychiatry 55:367-375.

Levine S (1994): The ontogeny of the hypothalamic-pituitary-adrenal axis. The influence of maternal factors. Ann NY Acad Sci 746: 275-288.

McCormick JA, Lyons V, Jacobson MD, Noble J, Diorio J, Nyirenda M, Weaver S, Ester W, Yau JLW, Meaney MJ, Seckl JR, Chapman KE (2000): 5'-Heterogeneity of glucocorticoid receptor messenger RNA is tissue specific: differential regulation of variant transcripts by early life events. Mol Endocrionol 14:506517.

- McCormick CM, Mathews IZ, Thomas C, Waters $\mathrm{P}$ (2010): Investigations of HPA function and the enduring consequences of stressors in adolescence in animal models. Brain Cogn 72:73-85.

Millstein RA, Holmes A (2007): Effects of repeated maternal separation on anxiety- and depression-related phenotypes in different mouse strains. Neurosci Biobehav Rev 31: 3-17.

-Mizoguchi K, Ishige A, Aburada M, Tabira T (2003): Chronic stress attenuates glucocorticoid negative feedback: involvement of the prefrontal cortex and hippocampus. Neuroscience 119:887-897. pressant stimulation of hippocampal neurogenesis is age-dependent and affected by early life stress. J Comp Neurol 509:372-381.

O’Donovan KJ, Tourtellotte WG, Millbrandt J, Baraban JM (1999): The EGR family of transcription-regulatory factors: progress at the interface of molecular and systems neuroscience. Trends Neurosci 22:167-173.

Plotzky PM, Meaney MJ (1993): Early, postnatal experience alters hypothalamic corticotropin-releasing factor (CRF) mRNA, median eminence CRF content and stress-induced release in adult rats. Brain Res Mol Brain Res 18:195-200.

Revest J-M, Di Blasi F, Kitchener P, Rougé-Pont F, Desmeddt A, Turiault M, Troche F, Piazza PV (2005): The MAPK pathway and egr-1 mediate stress-related behavioral effects of glucocorticoids. Nat Neurosci 8:664-672.

Ribeiro SC, Tandon R, Grunhaus L, Greden JF (1993): The DST as a predictor of outcome in depression: a meta-analysis. Am J Psychiatry 150:1618-1629.

Schmidt MV, Sterlemann V, Wagner K, Niederleitner B, Ganea K, Liebl C, Deussing JM, Berger S, Schütz G, Holsboer F, Müller MB (2009): Postnatal glucocorticoid excess due to pituitary glucocorticoid receptor deficiency: differential short- and long-term consequences. Endocrinology 150:2709-2716.

Weaver ICG, D’Alessio AC, Brown SE, Hellstrom IC, Dymov S, Sharma S, Szyf M, Meaney MJ (2007): The transcription factor nerve growth factor-inducible protein A mediates epigenetic programming: altering epigenetic marks by immediate-early genes. J Neurosci 27:1756-1768. 